\title{
A relativistic quark model with infrared confinement and the tetraquark state
}

\author{
S. Dubnicka* ${ }^{*}$ A.Z. Dubnickova ${ }^{\dagger}$, M.A. Ivanov** ${ }^{* *}$ J.G. Körner ${ }^{\ddagger}$ and \\ G.G. Saidullaeva ${ }^{\S}$ \\ *Institute of Physics Slovak Academy of Sciences Dubravska cesta 9 SK-842 28 Bratislava, Slovak Republic \\ ${ }^{\dagger}$ Comenius University Dept. of Theoretical Physics Mlynska Dolina SK-84848 Bratislava, Slovak Republic \\ ${ }^{* *}$ Bogoliubov Laboratory of Theoretical Physics, Joint Institute for Nuclear Research, 141980 Dubna, Russia \\ *Institut für Physik, Johannes Gutenberg-Universität, D-55099 Mainz, Germany \\ ${ }^{\S}$ Al-Farabi Kazak National University, 480012 Almaty, Kazakhstan
}

\begin{abstract}
We explore the consequences of treating the X(3872) meson as a tetraquark bound state. As dynamical framework we employ a relativistic constituent quark model which includes infrared confinement in an effective way. We calculate the decay widths of the observed channels $X \rightarrow J / \psi+2 \pi(3 \pi)$ and $X \rightarrow \bar{D}^{0}+D^{0}+\pi^{0}$ via the intermediate off-shell states $X \rightarrow J / \psi+\rho(\omega)$ and $X \rightarrow \bar{D}+D^{*}$. For reasonable values of the size parameter $\Lambda_{X}$ of the $\mathrm{X}(3872)$ we find consistency with the available experimental data.
\end{abstract}

Keywords: Relativistic quark model, confinement, exotic mesons PACS: $12.39 . \mathrm{Ki}, 13.25 . \mathrm{Ft}, 13.25 . \mathrm{Jx}, 14.40 . \mathrm{Rt}$

\section{INTRODUCTION}

A narrow charmonium-like state $X(3872)$ was observed in 2003 in the exclusive decay process $B^{ \pm} \rightarrow$ $K^{ \pm} \pi^{+} \pi^{-} J / \psi$ [1]. The $X(3872)$ decays into $\pi^{+} \pi^{-} J / \psi$ and has a mass of $m_{X}=3872.0 \pm 0.6$ (stat) \pm 0.5 (syst) very close to the $M_{D^{0}}+M_{D^{* 0}}=3871.81 \pm 0.25$ mass threshold [2]. Its width was found to be less than 2.3 $\mathrm{MeV}$ at $90 \%$ confidence level. The state was confirmed in B-decays by the BaBar experiment [3] and in $p \bar{p}$ production by the Tevatron experiments [4].

From the observation of the decay $X(3872) \rightarrow J / \psi \gamma$ reported by [5], it was shown that the only quantum numbers compatible with the data are $J^{P C}=1^{++}$or $2^{-+}$. However, the observation of the decays into $D^{0} \bar{D}^{0} \pi^{0}$ by the Belle and BaBar collaborations [6] allows one to exclude the choice $2^{-+}$because the near-threshold decay $X \rightarrow D^{0} \bar{D}^{0} \pi^{0}$ is expected to be strongly suppressed for $J=2$.

The Belle collaboration has reported evidence for the decay mode $X \rightarrow \pi^{+} \pi^{-} \pi^{0} J / \psi$ with a strong three-pion peak between $750 \mathrm{MeV}$ and the kinematic limit of 775 $\mathrm{MeV}$ [5], suggesting that the process is dominated by the sub-threshold decay $X \rightarrow \omega J / \psi$. It was found that the branching ratio of this mode is almost the same as that of the mode $X \rightarrow \pi^{+} \pi^{-} J / \psi$ :

$\frac{\mathscr{B}\left(X \rightarrow J / \psi \pi^{+} \pi^{-} \pi^{0}\right)}{\mathscr{B}\left(X \rightarrow J / \psi \pi^{+} \pi^{-}\right)}=1.0 \pm 0.4($ stat $) \pm 0.3$ (syst)

These observations imply strong isospin violation because the three-pion decay proceeds via an intermedi- ate $\omega$-meson with isospin 0 whereas the two-pion decay proceeds via the intermediate $\rho$-meson with isospin 1 . Also the two-pion decay via the intermediate $\rho$-meson is very difficult to explain by using an interpretation of the $X(3872)$ as a simple $c \bar{c}$ charmonium state with isospin 0 .

There are several different interpretations of the $X(3872)$ in the literature: a molecule bound state $\left(D^{0} \bar{D}^{* 0}\right)$ with small binding energy, a tetraquark state composed of a diquark and antidiquark, threshold cusps, hybrids and glueballs. A description of the current theoretical and experimental situation for the new charmonium states may be found in the reviews [7].

We provided in Ref. [8] an independent analysis of the the properties of the $X(3872)$ meson which we interpret as a tetraquark state as in [9]. We worked in the framework of the relativistic constituent quark model which has recently been extended to include infrared confinement effects [10].

\section{THEORETICAL FRAMEWORK}

The authors of [9] suggested to consider the $X(3872)$ meson as a $J^{P C}=1^{++}$tetraquark state with a symmetric spin distribution: $[c q]_{S=0}[\bar{c} \bar{q}]_{S=1}+[c q]_{S=1}[\bar{c} \bar{q}]_{S=0}$, $(q=u, d)$. The nonlocal version of the four-quark inter- 
polating current reads

$$
\begin{aligned}
& J_{X_{q}}^{\mu}(x)=\int d x_{1} \ldots \int d x_{4} \delta\left(x-\sum_{i=1}^{4} w_{i} x_{i}\right) \\
& \times \Phi_{X}\left(\sum_{i<j}\left(x_{i}-x_{j}\right)^{2}\right) \\
& \times \frac{1}{\sqrt{2}} \varepsilon_{a b c} \varepsilon_{d e c}\left\{\left[q_{a}\left(x_{4}\right) C \gamma^{5} c_{b}\left(x_{1}\right)\right]\left[\bar{q}_{d}\left(x_{3}\right) \gamma^{\mu} C \bar{c}_{e}\left(x_{2}\right)\right]\right. \\
& \left.+\left(\gamma^{5} \leftrightarrow \gamma^{\mu}\right)\right\}
\end{aligned}
$$

where $w_{1}=w_{2}=m_{c} / 2\left(m_{q}+m_{c}\right)$ and $w_{3}=w_{4}=$ $m_{q} / 2\left(m_{q}+m_{c}\right)$. The matrix $C=\gamma^{0} \gamma^{2}$ is the charge conjugation matrix. The effective interaction Lagrangian describing the coupling of the meson $X_{q}$ to its constituent quarks is written in the form

$$
\mathscr{L}_{\text {int }}=g_{X} X_{q \mu}(x) \cdot J_{X_{q}}^{\mu}(x), \quad(q=u, d) .
$$

The state $X_{u}$ breaks isospin symmetry maximally so the authors of [9] take the physical states to be a linear superposition of the $X_{u}$ and $X_{d}$ states according to

$$
\begin{aligned}
X_{l} \equiv X_{\text {low }} & =\quad X_{u} \cos \theta+X_{d} \sin \theta \\
X_{h} \equiv X_{\text {high }} & =\quad-X_{u} \sin \theta+X_{d} \cos \theta .
\end{aligned}
$$

The mixing angle $\theta$ can be determined from fitting the ratio of branching ratios Eq. (1).

The coupling constant $g_{X}$ in Eq. (3) will be determined from the compositeness condition $Z_{H}=0$, see, e.g. Refs. [11] and [12]. It gives

$$
Z_{X}=1-\Pi_{X}^{\prime}\left(m_{X}^{2}\right)=0,
$$

where $\Pi_{X}\left(p^{2}\right)$ is the scalar part of the vector-meson mass operator. The corresponding three-loop diagram describing the X-meson mass operator is shown in Fig. 1. We

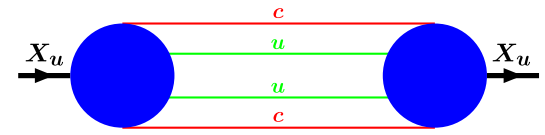

FIGURE 1. Diagram describing the $X_{u}$-meson mass operator.

will choose a simple Gaussian form for the vertex function with the only dimensional parameter $\Lambda_{X}$ characterizing the size of the $\mathrm{X}$-meson.

In [10] we described how to integrate $n$-point one-loop diagrams and how to implement infrared confinement of quarks in this process. We extend our loop integration techniques to the case of arbitrary number of loops. Let $n, \ell$ and $m$ be the number of the propagators, loops and vertices, respectively. In Minkowski space the $\ell$-loop diagram will be represented as

$$
\begin{aligned}
& \Pi\left(p_{1}, \ldots, p_{m}\right)= \\
= & \int\left[d^{4} k\right]^{\ell} \prod_{i_{1}=1}^{m} \Phi_{i_{1}+n}\left(-K_{i_{1}+n}^{2}\right) \prod_{i_{3}=1}^{n} S_{i_{3}}\left(\tilde{k}_{i_{3}}+v_{i_{3}}\right), \\
& K_{i_{1}+n}^{2}=\sum_{i_{2}}\left(\tilde{k}_{i_{1}+n}^{\left(i_{2}\right)}+v_{i_{1}+n}^{\left(i_{2}\right)}\right)^{2}
\end{aligned}
$$

where the vectors $\tilde{k}_{i}$ are linear combinations of the loop momenta $k_{i}$. The $v_{i}$ are linear combinations of the external momenta $p_{i}$ to be specified in the following. The strings of Dirac matrices appearing in the calculation need not concern us since they do not depend on the momenta. The external momenta $p_{i}$ are all chosen to be ingoing such that one has $\sum_{i=1}^{m} p_{i}=0$.

Using the Schwinger representation of the local quark propagator one has

$$
S(k)=(m+\not k) \int_{0}^{\infty} d \beta e^{-\beta\left(m^{2}-k^{2}\right)} .
$$

The integrand in Eq. (6) has a Gaussian form and may be integrated out explicitly. After doing the loop integrations one obtains

$$
\Pi=\int_{0}^{\infty} d^{n} \beta F\left(\beta_{1}, \ldots, \beta_{n}\right),
$$

where $F$ stands for the whole structure of a given diagram. The set of Schwinger parameters $\beta_{i}$ can be turned into a simplex by introducing an additional $t$-integration leading to

$$
\Pi=\int_{0}^{\infty} d t t^{n-1} \int_{0}^{1} d^{n} \alpha \delta\left(1-\sum_{i=1}^{n} \alpha_{i}\right) F\left(t \alpha_{1}, \ldots, t \alpha_{n}\right) .
$$

There are altogether $n$ numerical integrations: $(n-1) \alpha-$ parameter integrations and the integration over the scale parameter $t$. The very large $t$-region corresponds to the region where the singularities of the diagram with its local quark propagators start appearing. However, as described in [10], if one introduces an infrared cut-off on the upper limit of the t-integration, all singularities vanish because the integral is now convergent for any value of the set of kinematic variables. By introducing the infrared cut-off one has removed all potential thresholds in the quark loop diagram, i.e. the quarks are never on-shell and are thus effectively confined. We take the cut-off parameter $\lambda$ to be the same in all physical processes.

Next we evaluate the matrix elements of the transitions $X \rightarrow J / \psi+\rho(\omega)$ and $X \rightarrow D+\bar{D}^{*}$. The relevant Feynman diagrams are shown in Fig. 2. Since the $\mathrm{X}(3872)$ is 

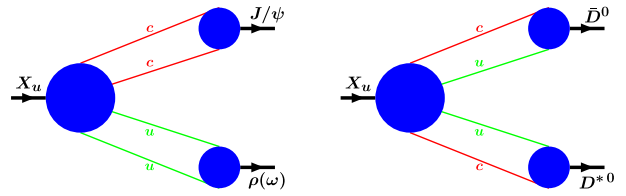

FIGURE 2. Feynman diagrams describing the decays $X \rightarrow$ $J / \psi+\rho(\omega)$ and $X \rightarrow D+\bar{D}^{*}$.

very close to the respective thresholds in both cases, the intermediate $\rho, \omega$ and $D^{*}$ mesons have to be treated as off-shell particles.

\section{NUMERICAL ANALYSIS}

Using the calculated matrix elements for the decay $X \rightarrow$ $J / \psi+\rho(\omega)$ one can evaluate the decay widths $X \rightarrow$ $J / \psi+2 \pi(3 \pi)$. We employ the narrow width approximation for this purpose.

The adjustable parameters of our model are the constituent quark masses $m_{q}$, the scale parameter $\lambda$ characterizing the infrared confinement and the size parameters $\Lambda_{M}$. They were determined by using a least square fit to a number of physical observables, see [10].

There are two new free parameters: the mixing angle $\theta$ in Eq. (4) and the size parameter $\Lambda_{X}$. We have varied the parameter $\Lambda_{X}$ in a large interval and found that the ratio

$$
\frac{\Gamma\left(X_{u} \rightarrow J / \psi+3 \pi\right)}{\Gamma\left(X_{u} \rightarrow J / \psi+2 \pi\right)} \approx 0.25
$$

is very stable under variations of $\Lambda_{X}$. Hence, by using this result and the central value of the experimental data given in Eq. (1), one finds $\theta \approx \pm 18.4^{\circ}$ for $X_{l}$ ("+") and $X_{h}$ ("-"), respectively. This is in agreement with the results obtained in both [9]: $\theta \approx \pm 20^{\circ}$ and [13]: $\theta \approx$ $\pm 23.5^{\circ}$. The decay width is quite sensitive to the change of the size parameter $\Lambda_{X}$. A natural choice is to take a value close to $\Lambda_{J / \psi}$ and $\Lambda_{\eta_{c}}$ which are both around 3 $\mathrm{GeV}$. We have varied the size parameter $\Lambda_{X}$ from 3 up to $4 \mathrm{GeV}$ and found that the decay width $\Gamma(X \rightarrow J / \psi+n \pi)$ decreases from 0.30 up to $0.07 \mathrm{MeV}$, monotonously. This result is in accordance with the experimental bound $\Gamma(X(3872)) \leq 2.3 \mathrm{MeV}$ and the result obtained in [9]: $1.6 \mathrm{MeV}$.

In a similar way we calculate the width of the decay $X \rightarrow D^{0} \bar{D}^{0} \pi^{0}$ which was observed by the Belle Coll. and reported in [6]. We have varied $\Lambda_{X}$ from 3 up to 4 $\mathrm{GeV}$ and found that the decay width $\Gamma\left(X_{l} \rightarrow \bar{D}^{0} D^{0} \pi^{0}\right)$ decreases from 1.88 up to $0.41 \mathrm{MeV}$, monotonously.

Using the results of [2], one calculates the experimental rate ratio

$$
\frac{\Gamma\left(X \rightarrow D^{0} \bar{D}^{0} \pi^{0}\right)}{\Gamma\left(X \rightarrow J / \psi \pi^{+} \pi^{-}\right)}=10.5 \pm 4.7
$$

The theoretical value for this rate ratio depends only weakly on the size parameter $\Lambda_{X}$

$$
\left.\frac{\Gamma\left(X \rightarrow D^{0} \bar{D}^{0} \pi^{0}\right)}{\Gamma\left(X \rightarrow J / \psi \pi^{+} \pi^{-}\right)}\right|_{\text {theor }}=6.0 \pm 0.2
$$

The theoretical error reflects the $\Lambda_{X}$ dependence of the ratio. The ratio lies within the experimental uncertainties given by Eq. (11).

\section{ACKNOWLEDGMENTS}

This work was supported by the DFG grant KO 1069/131, the Heisenberg-Landau program, the Slovak aimed project at JINR and the grant VEGA No.2/0009/10. M.A.I. also appreciates the partial support of the Russian Fund of Basic Research grant No. 10-02-00368-a.

\section{REFERENCES}

1. S. K. Choi et al. [Belle Collaboration], Phys. Rev. Lett. 91, 262001 (2003).

2. C. Amsler et al. [Particle Data Group], Phys. Lett. B 667, 1 (2008).

3. B. Aubert et al. [BaBar Collaboration], Phys. Rev. Lett. 93, 041801 (2004).

4. D. E. Acosta et al. [CDF Collaboration], Phys. Rev. Lett. 93, 072001 (2004); V. M. Abazov et al. [D0 Collaboration], Phys. Rev. Lett. 93, 162002 (2004); T. Aaltonen, et al. [CDF Collaboration], Phys. Rev. Lett. 103, 152001 (2009).

5. K. Abe et al., [Belle Collaboration], [arXiv:hepex/0505037,hep-ex/0505038]; B. Aubert et al. [BaBar Collaboration], Phys. Rev. D 74, 071101 (2006); A. Abulencia et al. [CDF Collaboration], Phys. Rev. Lett. 98, 132002 (2007).

6. G. Gokhroo et al. [Belle Collaboration], Phys. Rev. Lett. 97, 162002 (2006); B. Aubert et al. [BaBar Collaboration], Phys. Rev. D 77, 011102 (2008).

7. E. Eichten, S. Godfrey, H. Mahlke and J. L. Rosner, Rev. Mod. Phys. 80, 1161 (2008); S. Godfrey and S. L. Olsen, Ann. Rev. Nucl. Part. Sci. 58, 51 (2008); M. B. Voloshin, Prog. Part. Nucl. Phys. 61, 455 (2008).

8. S. Dubnicka, A. Z. Dubnickova, M. A. Ivanov and J. G. Körner, Phys. Rev. D81, 114007 (2010).

9. L. Maiani, F. Piccinini, A. D. Polosa and V. Riquer, Phys. Rev. D 71, 014028 (2005).

10. T. Branz, A. Faessler, T. Gutsche, M. A. Ivanov, J. G. Körner and V. E. Lyubovitskij, Phys. Rev. D 81, 034010 (2010).

11. G. V. Efimov, M. A. Ivanov, "The Quark confinement model of hadrons," Bristol, UK: IOP (1993) 177 p.

12. A. Salam, Nuovo Cim. 25, 224 (1962); S. Weinberg, Phys. Rev. 130, 776 (1963).

13. F. S. Navarra and M. Nielsen, Phys. Lett. B 639, 272 (2006). 